\title{
Filhos com Sintomas Psicológicos Clínicos: Papel Discriminante da Conjugalidade, Coparentalidade e Parentalidade
}

\author{
Clarisse Mosmann ${ }^{1}$ \\ Orcid.org/0000-0002-9275-1105 \\ Crístofer Batista da Costa ${ }^{1, *}$ \\ Orcid.org/0000-0002-1307-1436 \\ Allana Gessiele Mello da Silva ${ }^{1}$ \\ Orcid.org/0000-0002- 3086-2109 \\ Susana Konig $\mathrm{Luz}^{2}$ \\ Orcid.org/0000-0001-9508-1699 \\ ${ }^{2}$ Faculdade Meridional, Passo Fundo, RS, Brasil
}

${ }^{1}$ Universidade do Vale do Rio dos Sinos, São Leopoldo, RS, Brasil

\begin{abstract}
Resumo
Estudos indicam que a saúde mental dos filhos sofre reverberações positivas e negativas não somente da relação pais-filhos, mas também de aspectos da conjugalidade e da coparentalidade. Entretanto, a natureza e a magnitude dessas associações, quando analisadas em conjunto, ainda não foram suficientemente compreendidas, especialmente no contexto nacional. Nesse sentido, objetivou-se investigar em crianças e adolescentes com e sem sintomas psicológicos clínicos, qual o papel discriminante das variáveis da relação conjugal, coparental e parental de seus pais. A amostra foi composta por 200 participantes, com filhos de 4 a 18 anos, casados e coabitando com a prole. Através de análise estatística discriminante identificou-se as variáveis competição coparental, prática parental de intrusividade, exposição do filho ao conflito coparental e conflito conjugal como discriminante dos filhos com sintomas clínicos. Os resultados apontam que o subsistema coparental prepondera nesta relação, entretanto as três dimensões analisadas interagem de forma interdependente no ajustamento psicológico dos filhos.
\end{abstract}

Palavras-chave: Crianças, adolescentes, sintomas, relações familiares.

\section{Children with Clinical Psychological Symptoms: The Discriminant Role of Conjugality, Coparenting and Parenting}

\begin{abstract}
Studies indicate that the mental health of children suffer positive and negative reverberations not only from the parent-child relationship, but also from marital and coparenting aspects. However, the nature and magnitude of these associations, when considered together, are not yet sufficiently understood,
\end{abstract}

* Endereço para correspondência: Universidade do Vale do Rio dos Sinos, Programa de Pós-Graduação em Psicologia, Av. Unisinos, 950, São Leopoldo, RS, Brasil 93022-000. Fone: (51) 3591-1207. E-mail: cristoferbatistadacosta@gmail.com e allanagmsilva@gmail.com 
especially in the national context. This study aims to investigate in children and adolescents with and without clinical psychological symptoms, the discriminant role of the variables of their parent's marital, parental and coparental relations. The sample consists of 200 participants, with children 4-18 years old in a steady relationship and cohabiting with the offspring. Through discriminating statistical analysis the variables coparental competition, intrusiveness of parenting, child exposure to coparenting conflict, and marital conflict were identified as discriminant of children with clinical symptoms. The results indicate that the coparental subsystem prevails in this relationship, however the three dimensions analyzed interact interdependently in the psychological adjustment of children.

Keywords: Children, adolescents, symptoms, family relations.

\section{Hijos con Síntomas Psicológicos Clínicos: El Rol Discriminante de la Conyugalidad, Coparentalidad y Parentalidad}

\section{Resumen}

La salud mental de niños sufre repercusiones positivas y negativas no sólo de la relación padres e hijos, sino también del matrimonio y de la coparentalidad. Sin embargo, la naturaleza y magnitud de estas asociaciones, cuando se consideran conjuntamente, no están aun suficientemente comprendidas, especialmente en el contexto nacional. Se objetivó investigar en niños y adolescentes con y sin síntomas psicológicos clínicos, el rol discriminante de las variables de la relación matrimonial, parental y coparental. La muestra fueron 200 participantes, con niños de 4-18 años, en relación estable y que cohabitan con estos. El análisis discriminante mostró las variables competencia coparental, práctica parental de intrusión, exposición del niño al conflicto coparental y el conflicto conyugal como discriminantes de los niños con síntomas clínicos. Los resultados indican que el subsistema coparental prevalece en esta relación, sin embargo, las tres dimensiones analizadas interactúan de manera interdependiente en el ajuste psicológico de niños.

Palabras clave: Niños, adolescentes, síntomas, relaciones familiares.

Os estudos sobre as repercussões das características do funcionamento familiar no desenvolvimento infantil evoluíram de forma consistente nas duas últimas décadas. Percebe-se que a saúde mental e o comportamento dos filhos sofrem reverberações positivas e negativas não somente da relação pais-filhos, mas também de aspectos da conjugalidade dos pais e da coparentalidade (Einsfeld, Silva, Trindade, \& Mosmann, 2012; Mosmann, Costa, Einsfeld, Silva, \& Koch, 2017; Silva \& Mosmann, 2014).

A coparentalidade é definida por Feinberg (2003) como o envolvimento conjunto e recíproco dos pais na educação, formação e decisões sobre a vida dos seus filhos. No entanto, trata-se de um construto complexo que não está restrito somente ao pai e a mãe, considerando-se a relação entre pelo menos duas pessoas que assumem, por mútuo acordo ou pelas normas sociais, a responsabilidade conjunta pelo bem-estar de pelo menos uma criança (Marsanic \& Kusmic, 2013). Em famílias nucleares, que não passaram pelo divórcio dos pais, a coparentalidade é dinamicamente interligada com outros subsistemas familiares, mais concretamente com a conjugalidade e a parentalidade (Lamela, Nunes-Costa, \& Figueiredo, 2010).

As pesquisas sobre a reverberação das características do funcionamento familiar no desenvolvimento da prole focalizam principalmente os processos relacionais entre a conjugalidade, a parentalidade e a coparentalidade com o objetivo de desvelar as variáveis específicas que engendram os mecanismos de interação entre esses subsistemas (Morril, Hines, Mahmood, \& Cordova, 2010). Este esforço se fundamenta na tentativa de identificar com maior precisão o peso e o papel de cada subsistema familiar nesta interação, para se obter subsídios a promoção de relações conjugais com melhores níveis de qualida- 
de. Estas que atuarão como fonte de apoio para os cônjuges em relação a parentalidade, a coparentalidade e, por conseguinte, de proteção para o desenvolvimento dos filhos (Mosmann, Wagner, \& Sarriera, 2008; Wagner \& Mosmann, 2009).

Compreende-se que a qualidade da relação conjugal provoca reflexos na forma como os pais gerenciarão os problemas com as crianças, pois a habilidade para resolver os conflitos conjugais se reflete no exercício da parentalidade. Tal compreensão alude ao conceito Spillover, (Erel \& Burman, 1995) sobre os efeitos das relações conjugais transbordarem para a parentalidade (Merrifield \& Gamble, 2012), influenciando negativa ou positivamente o desenvolvimento dos filhos e, portanto, configurando-se em fator de risco ou proteção à criança (Christopher, Umemura, Mann, Jacobvitz, \& Hazen, 2015; Mosmann et al., 2008; Schmidt, Crepaldi, Vieira, \& Moré, 2011).

Nesse sentido, uma pesquisa realizada nos EUA investigou 149 famílias com filhos entre 3 e 6 anos e comprovou a hipótese Spillover (Stroud, Wilson, Durbin, \& Mendelsohn, 2011). O transbordamento ocorre em múltiplos subsistemas familiares, mas os efeitos variam de acordo com a forma como o comportamento mãe-pai-filho é explorado. Os principais resultados da pesquisa apontaram que o subsistema conjugal influenciou o afeto nas interações triádicas, bem como, nas respostas dos pais e das crianças nas interações diádicas em relação à mãe. Os efeitos do transbordamento foram equivalentes para meninos e meninas, mas o transbordamento para hostilidade parental nas interações triádicas foi limitado às famílias que educam meninas.

O sexo dos pais também influenciou as associações entre o funcionamento conjugal e as interações pai e filho. O transbordamento da qualidade conjugal ocorreu numa resposta afetiva dos pais e de uma resposta afetiva da criança às suas mães, mas não como resposta das mães ou da criança aos pais. Os efeitos do transbordamento foram mais significativos para a resposta dos pais, se comparado com a resposta das mães e mais forte para a resposta das crianças às mães, se comparado à resposta das crianças aos pais (Stroud et al., 2011).
Somado a isso, as práticas educativas parentais estão entre as principais variáveis associadas ao desenvolvimento socioemocional infantil, às relações entre pais e filhos e ao equilíbrio do ambiente familiar ao longo das diferentes etapas do ciclo vital da família. No entanto, ainda é impreciso o que se sabe sobre os fatores que interferem mais significativamente nas práticas educativas parentais. As questões comumente apontadas são: a ordem de nascimento e o número de filhos em uma família, a personalidade e o bem estar psicológico dos progenitores, a qualidade da relação conjugal, as experiências infantis vividas pelos pais, as questões de gênero, o nível de instrução dos pais, as características e comportamentos da criança, o contexto social, o momento do ciclo vital familiar e a própria divisão das práticas parentais entre o casal (Bolsoni-Silva, Paiva, \& Barbosa, 2009; Freitas \& Piccinini, 2010; Laxman et al., 2013; Teixeira, Oliveira, \& Wottrich, 2006).

Apesar de se confirmar empiricamente a substancial relação entre as variáveis citadas, não existe uma compreensão consensual acerca da natureza e magnitude dessas conexões. Se aceita que as relações não são de causa-efeito e que não podem ser reduzidas a um número pequeno de variáveis (Zahn-Waxler, Shirtcliff, \& Marceau, 2008). No entanto, é necessário definir quais serão as variáveis analisadas em cada estudo a fim de identificar aquelas que apresentam maior poder preditivo para o ajustamento dos filhos.

Quando o foco investigativo está no casal, percebe-se, por exemplo, que o conflito e a intimidade conjugal são variáveis que interferem no exercício da parentalidade (Engle \& McElwain, 2013), e, consequentemente, na negociação parento filial e no acordo coparental. Novamente a interação da dupla, enquanto pais, pode ser um fator determinante à proteção ou exposição do ambiente familiar (Portugal \& Isabel, 2013). Percebe-se, que existem inúmeras conexões e variáveis atuando entre os subsistemas familiares e cabe aos pesquisadores optar, segundo as evidências empíricas e o objetivo do estudo, por aquelas que poderão contribuir a explicação do fenômeno. 
Em uma pesquisa com 551 pais e mães americanos, as relações entre o conflito conjugal, a parentalidade e o ajustamento dos filhos foi objeto de investigação (Gerard, Krishnakumar, \& Buheler, 2006). Os resultados indicaram associação entre o conflito conjugal e a hostilidade parental, bem como, entre os baixos níveis de conflito e os altos níveis de envolvimento parental, apontando que a prole sente os reflexos das dimensões negativas e positivas da conjugalidade.

Especificamente, o estudo identificou que os genitores com altos níveis de conflito conjugal parecem ter menos tolerância para lidar com os desafios que as crianças trazem para o sistema familiar no dia-a-dia. Essa frustração aparece na falta de paciência dos pais com o comportamento dos filhos e com suas demandas, o que se traduz em baixa responsividade e estratégias educativas baseadas em controle coercitivo. Essa dinâmica reverbera longitudinalmente em problemas de externalização dos filhos (Gerard et al., 2006). De forma semelhante, nos casais que apresentam altos níveis de conflito conjugal, baixa responsividade e também baixa exigência, haverá um contexto fortemente preditor de problemas de internalização (Davies, Harold, Goeke-Morey, \& Cummings, 2002).

Nessa direção, Mosmann et al. (2008) confirmaram o valor preditivo das características positivas e negativas de cada cônjuge sobre os subsistemas conjugal e parental bem como sobre a dinâmica familiar. Foram avaliados 149 casais com filhos adolescentes traçando um perfil discriminante entre variáveis da relação conjugal e os estilos educativos parentais. Comprovou-se que os casais com maiores níveis de coesão, adaptabilidade e satisfação conjugal caracterizam o estilo educativo parental responsivo, marcado pela proximidade afetiva. As variáveis sexo, carga horária de trabalho e conflito/agressão discriminaram os casais em relação aos estilos educativos baixos em responsividade e as variáveis renda pessoal e tempo que passa com o filho, discriminaram respectivamente os estilos educativos negligente e autoritário.

A inter-relação com a conjugalidade, a parentalidade e o desenvolvimento infantil foi o foco de uma revisão sistemática em que foram analisadas publicações científicas nacionais e internacionais entre os anos de 1980 e 2010 (Schmidt et al., 2011). Os resultados indicaram associação significativa entre o temperamento infantil e a conjugalidade. Os maiores níveis de qualidade e ajustamento conjugal predisseram parceria nas práticas educativas, mesmo quando o temperamento infantil foi caracterizado como difícil pelos pais. De modo geral, os pesquisadores referem resultados associados, porém, em dois sentidos: reflexos negativos da baixa qualidade conjugal, do conflito e da parentalidade no desenvolvimento dos filhos assim como temperamento infantil difícil, como aspecto que prejudica a relação conjugal e as práticas parentais, indicando o caráter bidirecional dessas associações.

Neste sentido, sem considerar variáveis da relação conjugal, Bolsoni-Silva et al. (2009) apontam que as práticas parentais e o comportamento dos filhos se influenciam mutuamente e determinam, ao longo do tempo, que tipo de interação predominará entre a criança e os cuidadores. Segundo os autores, as práticas parentais coercitivas induzem respostas também negativas por parte dos filhos e o surgimento de sintomas externalizantes e internalizantes que, por sua vez, causam estresse e frustração aos pais.

Além das pesquisas e discussões acerca das repercussões da conjugalidade nas práticas parentais, no desenvolvimento infantil e na dinâmica familiar, as investigações sobre o subsistema coparental significaram avanços na área (Blandon, Scrimgeour, Stifter, \& Buss, 2014; Christopher et al., 2015; Holland \& McElwain, 2013; Laxman et al., 2013; Merrifield \& Gamble, 2012; Pedro, Ribeiro, \& Shelton, 2012; Riina \& McHale, 2015). Na literatura nacional há um esforço para se compreender a coparentalidade no contexto do divórcio (Lamela, Figueiredo, \& Bastos, 2013), porém, em famílias nucleares tem sido um assunto pouco explorado, apesar de já ter sido comprovado que a coparentalidade pode ser um elo de extrema relevância na associação entre a conjugalidade e a parentalidade (Einsfeld et al., 2012; Mosmann et al., 2017; Silva \& Mosmann, 2014). Isto porque é um componente 
único do relacionamento conjugal, considerando que os pais podem trabalhar de forma cooperativa ou travar uma batalha interminável, no que concerne às práticas educativas (Feinberg, 2003).

Pesquisas internacionais têm comprovado que o desenvolvimento das crianças poderia ser melhor explicado se agregarmos à análise também da coparentalidade nestas associações, considerando seu impacto nas relações sistêmicas familiares, do que pela qualidade conjugal e parental sozinhas (Kwon, Jeon, \& Elicker, 2013; Mendez, Loker, Fefer, Wolgemuth, \& Mann, 2015; Scrimgeour, Blandon, Stifter, \& Buss, 2013; Silva \& Mosmann, 2014) e que a coparentalidade é um fator relevante no desenvolvimento dos filhos, desde a infância até a adolescência (Christopher et al., 2015; Laxman et al., 2013; Mendez et al., 2015). Investigações da área indicam a necessidade de estudos que analisem se a coparentalidade funcionaria como mediadora em longo prazo entre aspectos negativos da relação conjugal, como baixos níveis de coesão e ajustamento, altos níveis de conflito e não resolução dos mesmos e problemas no desenvolvimento infantil (Jia, Kotila, \& Schoppe-Sullivan, 2012; Sbicigo \& Dell'Aglio, 2012).

Ademais, há evidências empíricas longitudinais de que a coparentalidade e o funcionamento familiar se associam ao desenvolvimento dos filhos em diferentes faixas etárias (Christopher et al., 2015; Laxman et al., 2013; Riina \& McHale, 2013). De forma geral, os indícios apontam que o comportamento dos filhos é afetado não somente pelas relações pais-filhos, mas também pela coparentalidade, quando os cônjuges falham no suporte um ao outro e expressam aos filhos práticas educativas contraditórias.

A literatura encontrada aponta variáveis dos três subsistemas, conjugal, parental e coparental, como atuantes na constituição de um sistema familiar protetivo para o desenvolvimento saudável dos filhos. Porém, para se avançar em termos de conhecimento dessa temática no contexto nacional é relevante a avaliação conjunta das três dimensões, conjugalidade, parentalidade e coparentalidade (Majdandzic, Vente, Feinberg, Aktar, \& Bögels, 2012) considerando que as pesquisas apontam resultados da análise das variáveis desses três subsistemas de forma independente.

Por isso, objetivou-se investigar em crianças e adolescentes com e sem sintomas psicológicos clínicos, qual o papel discriminante das variáveis da relação conjugal, parental e coparental de seus pais. As hipóteses norteadoras foram de que as variáveis positivas da coparentalidade (acordo; suporte; divisão de trabalho e aprovação coparental) teriam maior poder discriminante do grupo não clínico em relação às práticas parentais positivas (supervisão de comportamento; prática de apoio emocional/afeto; incentivo à autonomia; cobrança de responsabilidade) e a coesão e adaptabilidade conjugal (Christopher et al., 2015; Laxman et al., 2013; Riina \& McHale, 2013).Também estimou-se que as variáveis negativas da coparentalidade (competição e exposição do filho ao conflito coparental) teriam maior poder discriminante do grupo clínico em relação ao conflito conjugal e as práticas parentais negativas (intrusividade e controle punitivo; Jia et al., 2012; Sbicigo \& Dell'Aglio, 2012).

\section{Método}

\section{Delineamento}

Este é um estudo quantitativo de caráter exploratório, descritivo, comparativo e transversal.

\section{Amostra}

Participaram da pesquisa 200 indivíduos (100 homens e 100 mulheres). O cálculo amostral foi definido com base na recomendação de Hair, Anderson, Tatham, e Black, (2005) para realização de análise discriminante. Os autores definem 5 observações por variável independente, em nosso estudo temos 15 variáveis independentes, compreendendo um número mínimo de 75 indivíduos. Além disso, o tamanho de cada grupo deve ter no mínimo 20 observações. Nosso grupo clínico tem 20 filhos e o não-clínico, 180. Os critérios de inclusão foram homens e mulheres com pelo menos um filho com idade entre 4 e 18 anos, que estivessem casados ou vi- 
vendo em união estável e coabitassem com o filho, residentes na região metropolitana e interior do estado do Rio Grande do Sul.

\section{Instrumentos}

Dados Sócio Demográficos. Composto por treze perguntas fechadas que permitiram o levantamento dos dados sócio demográficos dos participantes da pesquisa.

Escala de Avaliação da Coesão e Adaptabilidade Conjugal - Faces III (Olson, 1986, validado por Falceto, 1997). Escala com vinte itens pontuados em uma escala Likert de cinco pontos (quase nunca, alguma vez, às vezes, com frequência, quase sempre). O coeficiente Alpha de Cronbach encontrado em outros estudos (Felgosa, 2013; Mosmann et al., 2008) foram considerados satisfatórios, sendo 0,80 e 0,78 para coesão e 0,67 e 0,72 para adaptabilidade, respectivamente. No presente estudo os índices foram 0,68 para coesão e 0,83 para adaptabilidade.

Escala de Conflito Conjugal (Buehler \& Gerard, 2002, adaptada por Mosmann et al., 2008). Nove itens que são apresentados separadamente em duas sub-escalas. A primeira denominada "conflito-desentendimentos" possui 6 itens avaliados em uma escala Likert de 6 pontos (nunca, uma vez ao mês ou menos, diversas vezes ao mês, aproximadamente uma vez por semana, diversas vezes por semana, quase todos os dias) que se referem a frequência com que os sujeitos experimentaram desentendimentos com seus parceiros no último ano. A outra sub-escala denominada "conflito-agressão" possui três itens pontuados em uma escala Likert de cinco pontos (nunca, raramente, algumas vezes, frequentemente, sempre). Na adaptação da escala (Mosmann et al., 2008) encontrou-se um coeficiente Alpha de Cronbach de 0,71 e de 0,78 neste estudo.

Parentalidade. Escala de práticas parentais, desenvolvida por Teixeira et al. (2006). A escala é composta de 27 itens e seis dimensões: apoio emocional, controle punitivo, incentivo à autonomia, intrusividade, supervisão do comportamento e cobrança de responsabilidade as quais são medidas em uma escala Likert de cinco pontos variando de "quase nunca ou bem pouco" a "geralmente ou bastante". No es- tudo original e no presente estudo, os índices de consistência interna encontrados foram, respectivamente, Alpha de Cronbach 0,89 e 0,89 (apoio emocional); 0,78 e 0,74 (controle punitivo); 0,76 e 0,65 (incentivo à autonomia); 0,67 e 0,63 (intrusividade); 0,77 e 0,77 (supervisão do comportamento); 0,70 e 0,61 (cobrança de responsabilidade).

Escala de Relação Coparental - ERC (Feinberg, Brawn, \& Kan, 2012, traduzida e adaptada para o presente estudo). A escala é composta de 35 itens que são apresentados em duas sub-escalas separadas e mensuram sete dimensões da coparentalidade: acordo coparental, proximidade coparental, exposição ao conflito coparental, suporte coparental, competição coparental, aprovação da parentalidade do outro e divisão do trabalho coparental. Os itens são medidos em uma escala do tipo Likert de seis pontos variando na primeira sub-escala de "não é verdadeiro sobre nós" até "muito verdadeiro sobre nós" e na segunda entre "nunca" e "muito frequente". Os índices de consistência interna encontrados no estudo original e neste estudo foram, respectivamente, Alpha de Cronbach 0,74 e 0,68 (acordo coparental); 0,81 e 0,47 (proximidade coparental); 0,89 e 0,78 (exposição ao conflito); 0,87 e 0,81 (suporte coparental), 0,85 e 0,85 (competição coparental); 0,83 e 0,63 (aprovação da parentalidade do outro). A dimensão divisão do trabalho tem apenas dois itens e por isto não pode ser mensurado o coeficiente de consistência interna. Considerando a baixa consistência interna da dimensão proximidade coparental esta foi excluída deste estudo.

Child Behavior Checklist - CBCL (Achenbach \& Rescorla, 2001, Alpha de Cronbach 0,97 para consistência interna total da escala). Esse instrumento apresenta evidências preliminares de validade para a população brasileira (Rocha et al., 2013). Escala que avalia os problemas emocionais e de comportamento dos filhos composta de 138 itens, destinado aos pais/mães ou cuidadores. Do total de itens, 20 são destinados à avaliação da competência social da criança e 118 relativos à avaliação de seus problemas de comportamento. Os itens do questionário listam uma série de comportamentos desejáveis e 
disruptivos em uma escala Likert de três pontos sendo 0 quando não é verdadeiro, 1 , se é um pouco verdadeiro ou às vezes verdadeiro e 2 , se é muito verdadeiro ou frequentemente verdadeiro. Os índices de consistência interna encontrados foram: ansiedade/depressão $(0,77)$ retraimento $(0,71)$ queixas somáticas $(0,67)$ problemas sociais $(0,72)$ comportamento delinquente $(0,73)$ problemas no pensamento $(0,66)$ comportamento agressivo $(0,82)$ problemas de atenção $(0,82)$ outros problemas $(0,61)$.

\section{Procedimentos}

Coleta de Dados. A amostra foi constituída por meio de coleta por conveniência, ou seja, pessoas da população acessíveis e disponíveis para participar do estudo. Estes participantes foram indicados por pessoas conhecidas dos membros do grupo de pesquisa, estes últimos que foram treinados para a coleta de dados. As pessoas indicadas foram contatadas por telefone e convidadas para participar do estudo. Diante do aceite, agendou-se dia e hora em que os membros do grupo de pesquisa foram até a casa do respondente.

Análise dos Dados. Os dados foram analisados através do programa estatístico SPSS (versão 20), considerando o nível de significância de $5 \%(p \leq 0,05)$. Foram avaliadas as propriedades psicométricas (confiabilidade e validade convergente) de cada instrumento. Foram feitas análises descritivas (médias, desvio-padrão, porcentagens) dos resultados em geral. Para a estatística inferencial, previamente foram analisados os critérios de supostos paramétricos (tipo de variável, tamanho da amostra, normalidade). Após realizou-se análise discriminante a qual é utilizada quando a variável dependente é dicotômica, neste caso crianças e adolescentes com e sem sintomas psicológicos clínicos, e tem por objetivo compreender as diferenças entre os grupos e prever a probabilidade de os participantes ser direcionada para um ou outro grupo (Hair et al., 2005).

\section{Considerações Éticas}

Esta pesquisa foi submetida e aprovada pelo Comitê de Ética em Pesquisa da Universidade do Vale do Rio dos Sinos, sob Protocolo número 11/016. O Termo de Consentimento Livre e Esclarecido - TCLE, redigido segundo as recomendações da Resolução n ${ }^{\circ}$ 466/2012 do Conselho Nacional de Saúde e 16/2000 do Conselho Federal de Psicologia, foi lido aos participantes. Diante do consentimento, os termos foram assinados em duas vias, uma para os respondentes e outra para arquivo institucional. Ao término da aplicação, o envelope com o instrumento e o TCLE foi lacrado na frente dos participantes visando à confidencialidade dos dados.

\section{Resultados}

A caracterização da amostra foi realizada por meio de análises descritivas. A idade média dos participantes foi 41,81 anos $(D P=7,82)$, sendo a idade mínima de 22 anos e a máxima 66 anos. Dos respondentes $81,5 \%$ eram casados e $18 \%$ viviam em união estável. Destes, $91 \%$ estavam em primeira união e $8 \%$ eram recasados. $\mathrm{O}$ tempo mínimo de união foi de 4 anos e o máximo de 36 anos, com tempo médio de 18,26 anos $(D P=6,68)$.

Em relação a escolaridade, $22,4 \%$ dos participantes tinham ensino fundamental completo/incompleto, $31,6 \%$ ensino médio completo/ incompleto, $6,6 \%$ ensino técnico, $28,1 \%$ ensino superior completo/incompleto e $11,2 \%$ pós-graduação. De todos os respondentes, $88 \%$ afirmaram exercer atividade remunerada, sendo que sua renda pessoal categorizada em seis grupos, até 2 salários mínimos foram $21,6 \%$, de 2 a 4 salários mínimos $28,6 \%$, de 4 a 6 salários mínimos $15,6 \%$, de 6 a 8 salários mínimos $8,5 \%$, de 8 a 10 salários mínimos $6 \%$ e acima de 10 salários mínimos $6 \%$ da amostra. A média do número de filhos dos participantes foi de 1,66 filhos (DP $=0,70$ ), sendo que $91,5 \%$ possuem entre um e dois filhos. O filho sobre o qual os participantes responderam os questionários tem idade média de 11,3 anos $(D P=4,25)$, sendo $59,5 \%$ do sexo masculino e $40,5 \%$ do sexo feminino.

Com o objetivo de identificar quais, dentre as 15 variáveis, que são as dimensões das escalas que compõem o estudo, discriminam as crianças e adolescentes do grupo clínico e do grupo não 
clínico procedeu-se à análise discriminante. Os escores de problemas de comportamento foram classificados na faixa não clínica (inferiores a 60) ou clínica (superiores a 60), conforme os autores do instrumento recomendam para fins de pesquisa (Achenbach \& Rescorla, 2001). Com base nesta classificação, os resultados indicam que $10 \%$ das crianças e adolescentes da nossa amostra são consideradas casos clínicos.

Em relação às variáveis testadas, estas foram: conjugalidade (conflito, coesão e adaptabilidade conjugal); coparentalidade (competição, exposição do filho ao conflito coparental, acordo, suporte, divisão de trabalho e aprovação coparental); parentalidade: (prática parental de incentivo à autonomia, prática de apoio emo- cional/afeto, prática parental de intrusividade, prática parental de cobrança de responsabilidade, prática parental de controle punitivo, prática parental de supervisão de comportamento).

Conforme os resultados apresentados na tabela 2, o ponto de corte estabelecido para as variáveis de maior poder explicativo foi de $\geq$ 0,3 (Sarriera et al., 2012). Em relação ao grupo discriminado, os valores do centróide foram de $(F=1,430)$ para as crianças e adolescentes com sintomas clínicos e $(F=-0,163)$ para as crianças e adolescentes sem sintomas. Os valores do centróide indicam que os grupos estão bem afastados do perfil obtido, sendo discriminados pelas variáveis apresentadas. Nas correlações obtidas a proximidade do centróide indica a direção favorável a um grupo ou outro.

Tabela 1

Valores da Função

\begin{tabular}{|c|c|c|c|c|c|c|c|c|}
\hline Função & $\begin{array}{l}\text { Valor } \\
\text { próprio }\end{array}$ & $\begin{array}{c}\% \text { da } \\
\text { variância }\end{array}$ & $\begin{array}{c}\text { Variância } \\
\text { Cumulativa }\end{array}$ & $\begin{array}{c}\text { Correlação } \\
\text { Canônica }\end{array}$ & $\lambda$ Wilks & $X^{2}$ & $g l$ & Sig. \\
\hline 1 & 0,235 & 100,0 & 100,0 & 0,436 & 0,810 & 37,291 & 15 & 0,001 \\
\hline
\end{tabular}

As variáveis que serão apresentadas ao longo dessa sessão estão ordenadas segundo o seu poder explicativo para a análise discriminante; pelo quanto são capazes de diferenciar o grupo clínico e não clínico, sendo que estas tiveram percentual de explicação de $80,6 \%$ dos dois grupos. Os resultados da análise apontaram que entre as 15 variáveis, 10 apresentaram maior magnitude (ponto de corte: valor da carga $\geq 0,3$ ) na discriminação dos grupos. Através da tabela 2 percebe-se que as variáveis competição coparental $(0,721)$, prática parental de intrusividade $(0,542)$, exposição do filho ao conflito coparental $(0,525)$ e conflito conjugal $(0,401)$ discriminaram mais fortemente os filhos com sintomas clínicos.

Tabela 2

Matriz de Estruturas

\begin{tabular}{|c|c|}
\hline Variáveis & Função 1 \\
\hline Competição coparental & 0,721 \\
\hline Acordo coparental & $-0,679$ \\
\hline Prática parental de intrusividade & 0,542 \\
\hline Exposição do filho ao conflito coparental & 0,525 \\
\hline Adaptabilidade conjugal & $-0,492$ \\
\hline Prática parental de incentivo a autonomia & $-0,478$ \\
\hline Divisão de trabalho coparental & $-0,473$ \\
\hline Conflito conjugal & 0,401 \\
\hline Suporte coparental & $-0,387$ \\
\hline Coesão conjugal & $-0,369$ \\
\hline
\end{tabular}




\section{Discussão}

De acordo com os resultados, identifica-se que a variável competição coparental apresentou maior grau de discriminação, sendo o principal fator da relação do par parental que reverbera no comportamento do filho. Esse resultado pode indicar que a disputa de forças ou a ausência de negociação acerca da responsabilidade partilhada entre o casal parental e das necessidades do filho provoca impacto negativo no comportamento do mesmo. Tal resultado reforça o que vem sendo apontado nas pesquisas (Blandon et al., 2014; Christopher et al., 2015; Sbicigo \& Dell'Aglio, 2012) acerca do quão significativo são os reflexos do subsistema coparental no desenvolvimento dos filhos.

As variáveis prática parental de intrusividade e exposição do filho ao conflito coparental tiveram poder de explicação discriminante semelhante e igualmente significativos. Nesse sentido, o exercício da parentalidade de forma intrusiva e a imposição de normas e comportamentos sem que haja diálogo é um método que pode não proporcionar a autonomia necessária à prole. Neste tipo de prática parental predominam os sentimentos de intolerância e o desrespeito que, associados à variável de exposição do filho ao conflito coparental, justificam seu poder de discriminação. Estas associações confirmam que o efeito Spillover (Erel \& Burman, 1995) postulado entre a conjugalidade e a parentalidade, pode ocorrer também entre a coparentalidade e a parentalidade nesta amostra, em que altos níveis de exposição do filho ao conflito coparental parecem se expressar em práticas parentais punitivas, o que por sua vez reverbera novamente em aumento nos índices de desacordo coparental e em sintomas psicológicos dos filhos (Gerard et al., 2006; Kwon et al., 2013; Stroud et al., 2011).

Tanto a prática parental de intrusividade quanto a exposição do filho ao conflito coparental conectam-se com a quarta variável que discrimina os filhos com sintomas clínicos. O conflito conjugal evidencia as dificuldades da díade para gerenciar e resolver os problemas com o parceiro de forma construtiva, fato que gera consequências na relação parento filial e reper- cute negativamente no desenvolvimento infantil através de sintomas psicológicos, conforme já postularam outros pesquisadores (Davies et al., 2002; Einsfeld et al., 2012; Gerard et al., 2006).

A competição coparental, a prática parental de intrusividade, a exposição do filho ao conflito coparental e o conflito conjugal discriminaram significativamente os filhos com sintomas clínicos. Esses resultados sustentam parcialmente nossa segunda hipótese, pois a dimensão com maior poder de discriminação efetivamente foi da coparentalidade, entretanto, ela foi seguida por dimensões da parentalidade e da conjugalidade. O poder de discriminação de cada variável suscita questionamentos.

Se o casal se constituiu sem filhos, o conflito conjugal inaugura os problemas familiares porque antecede à coparentalidade e à parentalidade. Estima-se então que seria protetivo ao ajustamento dos filhos que a capacidade para resolver os conflitos conjugais fosse desenvolvida antes do exercício da coparentalidade e da parentalidade (Mosmann et al., 2008). Nesse sentido, seria importante aos cônjuges identificar as situações de conflito que se mostram mais difíceis de manejar, buscando auxílio por meio de programas de educação conjugal ou ainda de psicoterapia individual, de casal ou familiar (Epstein, Warfel, Johnson, Smith, \& McKinney, 2013; Kumpfer \& Brooks, 2010). Tornar-se pais antes de aprender gerenciar construtivamente os conflitos que poderão, inclusive, aumentar em intensidade e frequência devido as dificuldades inerentes ao exercício da parentalidade pode ser um fator de risco ao desenvolvimento da prole. Isso pode ocorrer considerando que a criança nascerá em um contexto em que há conflitos que se somarão às demandas de cuidado e de reorganização do sistema familiar.

Por outro lado, as variáveis acordo coparental $(-0,679)$, adaptabilidade $(-0,492)$, prática parental de incentivo a autonomia $(-0,478)$, divisão de trabalho coparental $(-0,473)$, suporte coparental $(-0,387)$ e coesão conjugal $(-0,369)$ diferenciaram os filhos sem sintomas psicológicos clínicos. A variável acordo coparental teve o maior poder discriminatório. Este resultado aponta para os reflexos da negociação entre os pais 
acerca dos cuidados e responsabilidades com os filhos e sobre a capacidade destes últimos de identificar essa dinâmica no ambiente familiar (Mendez et al., 2015; Riina \& McHale, 2015). Essa evidência sustenta parcialmente nossa primeira hipótese e vai ao encontro do referido por Feinberg (2003) sobre o acordo coparental estar entre as principais estratégias mediadoras das relações entre pais e filhos e associadas a eficácia das práticas educativas, quando utilizadas pelos pais de forma homogênea.

A adaptabilidade foi a segunda variável que discriminou mais significativamente as crianças sem sintomas. Esse dado corrobora uma diversidade expressiva de estudos (Erel \& Burman, 1995; Gerard et al., 2006; Stroud et al., 2011; Wagner \& Mosmann, 2009) que atribuem a repercussão das variáveis da conjugalidade às principais demandas relacionadas ao desenvolvimento dos filhos, como fator de proteção. Assim como algumas variáveis atuam como fator de risco aos filhos neste estudo, a capacidade do casal de se adaptar as dificuldades do dia-a-dia, atua de forma protetiva ao desenvolvimento dos mesmos.

O resultado da variável prática parental de incentivo à autonomia, diferenciando os filhos sem sintomas difere, em certa medida, dos pressupostos de Freitas e Piccinini (2010) sobre as práticas educativas parentais se sobressaírem no que tange ao desenvolvimento socioemocional da criança e ao equilíbrio do contexto familiar durante as diferentes etapas do ciclo vital. Tal diferença pode estar relacionada a inserção, no presente estudo, do subsistema coparental, dado que nas evidências encontradas, variáveis desta dimensão discriminaram mais fortemente o grupo com e sem sintomas. Além disso, as variáveis divisão de trabalho coparental e suporte coparental indicaram que a capacidade do casal de partilhar as responsabilidades acerca da demanda do filho e apoiar o parceiro diante das circunstâncias imprevisíveis da parentalidade engendram um contexto relacional saudável para o desenvolvimento da prole (Scrimgeour et al., 2013).

A coesão é a última dimensão que diferenciou o grupo sem sintomas clínicos, compro- vando que a conexão emocional entre os cônjuges é um fator preditor de ajustamento aos filhos (Sbicigo \& Dell'Aglio, 2012) apontando para as conexões entre os subsistemas familiares (Erel \& Burman, 1995; Majdandzic et al., 2012). O casal que está próximo afetivamente também apresenta altos níveis de adaptabilidade, o que repercute em acordo e divisão do trabalho coparental e, em consequência, prática educativa de incentivo a autonomia dos filhos, os quais, por conseguinte apresentam ausência de sintomas psicológicos.

Destaca-se que nos dois grupos, com sintomas e sem sintomas, as dimensões antagônicas da coparentalidade, acordo e competição coparental, estiveram presentes com maior magnitude de discriminação, sustentando parcialmente nossas hipóteses e corroborando os achados de que este subsistema atua fortemente no desenvolvimento dos filhos positiva e negativamente (Feinberg, 2003; Lamela et al., 2010). Além disso, evidencia-se que casais que discordam, mas conseguem negociar as diferenças, conseguem gerenciar o acordo coparental.

Os resultados desta pesquisa indicam a interdependência entre os subsistemas. Nota-se que a conjugalidade, a coparentalidade e a parentalidade, através das variáveis que constituem cada uma das três dimensões, estiveram presentes nos dois grupos, filhos com e sem sintomas, discriminando-os a partir do ponto de corte definido para este estudo. Essa evidência refuta nossa hipótese de que a coparentalidade seria preponderante à conjugalidade e a parentalidade (Kwon et al., 2013; Mendez et al., 2015; Scrimgeour et al., 2013), mas reforça o que aponta a literatura (Zahn-Waxler et al., 2008) sobre a complexidade de um tema que envolve um número expressivo de variáveis interdependentes que interagem dinamicamente através de subsistemas (Morril et al., 2010; Schmidt et al., 2011) dentro do sistema familiar, gerando conexões de natureza e magnitude multideterminadas. Isso se evidencia ao verificar que dimensões dos diferentes subsistemas podem contribuir tanto positivamente, promovendo o desenvolvimento saudável dos filhos, quanto negativamente, associando-se ao desenvolvimento de sintomas. 


\section{Conclusões}

O objetivo deste estudo foi investigar em crianças e adolescentes com e sem sintomas psicológicos clínicos, qual o papel discriminante das variáveis da relação conjugal, parental e coparental de seus pais. Percebe-se que os três subsistemas familiares explorados no artigo, são interdependentes ao discriminarem os filhos com e sem sintomas psicológicos clínicos. Esta visão mais ampla do sistema familiar, que não enfoca somente a díade parental, ou a relação pai-filho/mãe-filho, torna-se necessária na sociedade ocidental, já que, atualmente, o envolvimento paterno vem cada vez mais sendo valorizado e a divisão de papéis se tornou mais flexível, demonstrando que a divisão do trabalho coparental impacta na dinâmica familiar. Desta forma, não se visualiza o sistema familiar somente como a soma das partes (subsistemas), mas observa-se a abrangência das complexas relações interacionais.

Os resultados também mostram as variáveis da coparentalidade com maior poder de discriminação entre os grupos, seguida das variáveis da parentalidade e da conjugalidade. Desta forma, compreende-se que a coparentalidade atua como um importante fator interveniente entre a conjugalidade e a parentalidade, pois reflete tanto na relação dos pais com a criança como dos cônjuges entre si, e ainda, através do transbordamento destes, conforme o conceito Spillover.

Nesta perspectiva, muitas contribuições podem ser feitas ao entendimento dos sintomas dos filhos quando se volta o olhar para o par parental. Uma das maneiras de sensibilizar o casal a entrar em contato com seus conflitos é através do sintoma dos seus filhos, que conforme apontam nossos dados, denuncia a dinâmica do casal. Esses sintomas constituem um meio auxiliar para os pais buscarem ajuda e se voltarem para o entendimento da dinâmica conjugal. Sugere-se, então, uma ampliação do olhar dos profissionais da área para o entendimento desses sintomas, englobando uma forma distinta de encaminhamento para o caso, onde toda a família receba atenção acreditando que esse tipo de interven- ção pode exercer um efeito não só curativo, mas também preventivo.

Nossos resultados constituem-se em evidências que podem subsidiar o foco dessas intervenções familiares, quais sejam: competição coparental, prática parental de intrusividade, exposição do filho ao conflito coparental e conflito conjugal. Nessa direção, estima-se que desenvolver habilidades de resolução de conflito entre os cônjuges, investindo na proteção dos filhos às suas dificuldades, potencializar a capacidade de suporte e divisão do trabalho coparental, assim como a coesão e adaptabilidade conjugal, tenderia a reverberar positivamente no ambiente familiar e em consequência na saúde mental da prole.

Como limitações identifica-se que a avaliação dos sintomas psicológicos das crianças e adolescentes baseou-se na percepção dos pais, assim como apenas através de auto relatos. Novas pesquisas, poderiam agregar a perspectiva dos filhos sobre estes fenômenos, assim como observações das interações familiares poderiam contribuir para um aprofundamento do conhecimento nesta área.

\section{Referências}

Achenbach, T. M., \& Rescorla, L. A. (2001). Manual for the Aseba School-Age Forms \& Profiles. Burlington, VT: University of Vermont, Research Center for Children, Youth \& Families.

Blandon, A. Y., Scrimgeour, M. B., Stifter, C. A., \& Buss, K. A. (2014). Within-and between-family differences in cooperative and competitive coparenting. Journal of Family Psychology, 28(1), 106-111. doi:10.1037/a0035266

Bolsoni-Silva, A. T., Paiva, M. M., \& Barbosa, C. G. (2009). Problemas de comportamento de crianças/adolescentes e dificuldades de pais/ cuidadores: Um estudo de caracterização. Psicologia Clínica, 21(1), 169-184. doi:10.1590/ S010356652009000100012

Buehler, C., \& Gerard, J. M. (2002). Marital conflict, ineffective parenting, and children's and adolescents' maladjustment. Journal of Marriage and Family, 64(1), 78-93. doi:10.1111/j.17413737.2002.00078.x

Christopher, C., Umemura, T., Mann, T., Jacobvitz, D., \& Hazen, N. (2015). Marital Quality over 
the Transition to Parenthood as a Predictor of Coparenting. Journal of Child and Family Studies, 24(12), 3636-3651. doi:10.1007/ s10826-015-0172-0

Davies, P. T., Harold, G. T, Goeke-Morey, M. C., \& Cummings, E. M. (2002). Child emotional security and interparental conflict. Monographs of the Society for Research in Child Development, 67(3), 1-115. Retrieved from http://www.jstor. org/stable/3181513

Einsfeld, P., Silva, A. G. M., Trindade, M. T., \& Mosmann, C. P. (2012). Interação conjugal, coparental, parental e sintomas internalizantes e externalizantes dos filhos. In II CICPG - Congresso de Iniciação Científica e Pós-Graduação (p. 639-640). São Leopoldo, RS.

Engle, J. M., \& McElwain, N. L. (2013). Parental depressive symptoms and marital intimacy at 4.5 years: Joint contributions to mothers-child and father-child interaction at 6.5 years. Developmental Psychology, 49(12), 2225-2235. doi: $10.1037 / \mathrm{a} 0032450$

Epstein, R., Warfel, R., Johnson, J., Smith, R., \& McKinney, P. (2013). Which relationship skills count most? Journal of Couple \& Relationship Therapy, 12(4), 297-313. doi:10.1080/1533269 1.2013 .836047

Erel, O., \& Burman, B. (1995). Interrelatedness of marital relations and parent-child relations: A metaanalytic review. Psychological Bulletin, 118(1), 108-132. doi:10.1037/0033-2909.118.1.108

Falceto, O. G. (1997). Famílias com desenvolvimento funcional e disfuncional: Validação das escalas diagnósticas Faces III, Beavers - Timberlawn e Avaliação Global do Funcionamento Interacional (Dissertação de mestrado, Programa de Pós-Graduação em Medicina: Clínica Médica, Universidade Federal do Rio Grande do Sul, Porto Alegre, RS, Brasil).

Felgosa, M. R. D. L. (2013). Coesão e adaptabilidade familiares: Estudo preliminar das características psicométricas da FACES III numa amostra de mães portuguesas (Dissertação de mestrado, Programa de Pós-Graduação em Psicologia Clínica e da Saúde, Universidade do Algarve, Portugal).

Feinberg, M. (2003). The internal structure and ecological context of coparenting: A framework for research and intervention. Parenting: Science and Practice, 3(2), 95-132. doi:10.1207/ S15327922PAR0302_01
Feinberg, M. E., Brown, L., \& Kan, M. L. (2012). A multi-domain, self-report measure of coparenting. Parenting: Science and Practice, 12(1), 1-21. doi:10.1080/15295192.2012.638870

Freitas, A. P. C. O., \& Piccinini, C. A. (2010). Práticas educativas parentais em relação ao filho único e ao primogênito. Estudos de Psicologia (Campinas), 27(4), 515-528. doi:10.1590/ S0103-166X2010000400009

Gerard, J. M., Krishnakumar, A., \& Buheler, C. (2006). Marital conflict, parent-child relations, and youth maladjustment a longitudinal investigation of spillover effects. Journal of Family Issues, 27(7), 951-975. doi:10.1177/0192513X05286020

Hair, J. F., Anderson, R. E., Tatham, R. L., \& Black, W. C. (2005). Análise Multivariada de Dados (5. ed.). Porto Alegre, RS: Artmed.

Holland, A. S., \& McElwain, N. L. (2013). Maternal and paternal perceptions of coparenting as a link between marital quality and the parent - Toddler relationship. Journal of Family Psychology, 27(1), 117-126. doi:10.1037/a0031427

Jia, R., Kotila, L. E., \& Schoppe-Sullivan, S. J. (2012). Transactional relations between father involvement and preschoolers' socioemotional adjustment. Journal of Family Psychology, 26(6), 848-857. doi:10.1037/a0030245

Kumpfer, K. L., \& Brooks, J. (2010). Effective family nurturing programs for the prevention of family violence and child maltreatment. In B. Fisher \& S. Lab (Eds.), Encyclopedia of Victimology and Crime Prevention. Thousand Oaks, CA: Sage.

Kwon, K. A., Jeon, H. J., \& Elicker, J. (2013). Links among coparenting quality, parental gentle guidance, and toddlers' social emotional competencies: Testing direct, mediational, and moderational models. Journal of Family Studies, 19(1), 19-34. doi:10.5172/ jfs.2013.19.1.19

Lamela, D., Figueiredo, B., \& Bastos, A. (2013). Perfis de vinculação, coparentalidade e ajustamento familiar em pais recém-divorciados: Diferenças no ajustamento psicológico. Psicologia: Reflexão e Crítica, 26(1), 19-28. doi:http://dx.doi. org/10.1590/S0102-79722013000100003

Lamela, D., Nunes-Costa, R., \& Figueiredo, B. (2010). Modelos teóricos das relações coparentais: Revisão crítica. Psicologia em Estudo, 15(1), 205216. doi:10.1590/S141373722010000100022 
Laxman, D. J., Jessee, A., Mangelsdorf, S. C., Rossmiller-Giesing, W., Brown, G. L., \& Schoppe-Sullivan, S. J. (2013). Stability and antecedents of coparenting quality: The role of parent personality and child temperament. Infant Behavior and Development, 36(2), 210-222. doi:10.1016/j. infbeh.2013.01.001

Majdandzic, M., Vente, W., Feinberg, M. E., Aktar, E., \& Bögels, S. M. (2012). Bidirectional associations between coparenting relations and family member anxiety: A review and conceptual model. Clinical Child and Family Psychology Review, 15(1), 28-42. doi:10.1007/s10567-0110103-6

Marsanic, B. V., \& Kusmic, E. (2013). Coparenting within the Family System: Review of Literature. Collegium Antropologicum, 37(4), 1379-1384.

Mendez, L. M. R., Loker, T., Fefer, S., Wolgemuth, J., \& Mann, A. (2015). "Either come together or fall apart": Coparenting young children with challenging behaviors. Couple and Family Psychology: Research and Practice, 4(2), 7491. doi:10.1037/cfp0000039

Merrifield, K. A., \& Gamble, W. C. (2012). Associations among marital qualities, supportive and undermining coparenting, and parenting self-efficacy: Testing spillover and stress-buffering processes. Journal of Family Issues, 34(4), 510 533. doi:10.1177/0192513X12445561

Morril, M. I., Hines, D. A., Mahmood, S., \& Cordova, J. V. (2010). Pathways between marriage and parenting for wives and husbands: The role of coparenting. Family Process, 49, 59-73. doi:10.1111/j.1545-5300.2010.01308.x

Mosmann, C. P., Costa, C. B., Einsfeld, P., Silva, A. G. M., \& Koch, C. (2017). Conjugalidade, parentalidade e coparentalidade: Associações com sintomas externalizantes e internalizantes em crianças e adolescentes. Estudos de Psicologia (Campinas), 34, 487-498.

Mosmann, C., Wagner, A., \& Sarriera, J. (2008). A qualidade conjugal como preditora dos estilos educativos parentais: $O$ perfil discriminante de casais com filhos adolescentes. Psicologia, 22(2), 161-182. Recuperado em http://revista. appsicologia.org/index.php/rpsicologia/article/ view/352

Olson, D. (1986). Circumplex Model VII: Validation studies and FACES III. Family Process, 25(3), 337-351. doi:10.1111/j.15455300.1986.00337.x
Pedro, M. F., Ribeiro, T., \& Shelton, K. H. (2012). Marital satisfaction and partners' parenting practices: The mediating role of coparenting behavior.Journal of Family Psychology, 26(4), 509-522. doi:10.1037/a0029121

Portugal, A., \& Isabel, A. M. (2013). A comunicação parento-filial: Estudo das dimensões comunicacionais realçadas por progenitores e por filhos. Psicologia: Reflexão e Crítica, 26(3), 479-487. doi:10.1590/S0102-79722013000300007

Riina, E. M., \& McHale, S. M. (2013). Bidirectional influences between dimensions of coparentig and adolescente adjustment. Journal of Youth Adolescence, 43(2), 257-269. doi:10.1007/ s10964-013-9940-6.

Riina, E. M., \& McHale, S. M. (2015). African american couples' coparenting satisfaction and marital characteristics in the first two decades of marriage. Journal of Family Issues, 36(7), 902-923. doi:10.1177/0192513X13495855

Rocha, M. M., Rescorla, L. A., Emerich D. R., Silvares, E. F. M., Borsa, J. C., Araújo, L. G. S., ...Assis, S. G. (2013). Behavioural/emotional problems in Brazilian children: Findings from parents' reports on the child behavior checklist. Epidemiology and Psychiatric Sciences, 22(4), 329-338, 1-10. doi:10.1017/ S2045796012000637

Sarriera, J. C., Ximenes, V. M., Bedin, L., Rodrigues, A. L., Schütz, F. F., Montserrat, C., ...Silva, C. L. (2012). Bem-estar pessoal de pais e filhos e seus valores aspirados. Aletheia, 37, 91-104. Recuperado em http://pepsic.bvsalud.org/pdf/ aletheia/n37/n37a07.pdf

Sbicigo, J. B., \& Dell'Aglio, D. D. (2012). Family environment and psychological adaptation in adolescents. Psicologia: Reflexão e Crítica, 25(3), 615-622.

Schmidt, B., Crepaldi, M. A., Vieira, M. L., \& Moré, C. L. O. O. (2011). Relacionamento conjugal e temperamento de crianças: Uma revisão da literatura. Arquivos Brasileiros de Psicologia, 63(3), 89-106. Recuperado em http://pepsic. bvsalud.org/pdf/arbp/v63n3/08.pdf

Scrimgeour, M. B., Blandon, A. Y., Stifter, C. A., \& Buss, K. A. (2013). Cooperative coparenting moderates the association between parenting practices and children's prosocial behavior. Journal of Family Psychology, 27(3), 506-511. doi:10.1037/a0032893 
Silva, A. G. M., \& Mosmann, C. (2014). Fatores conjugais, coparentais e parentais como preditores de sintomas internalizantes e externalizantes dos filhos. In XXI Mostra Unisinos de Iniciação Cientifica e Tecnológica. Anais da Mostra Unisinos de Iniciação Científica e Tecnológica (pp. 210-211). São Leopoldo, RS: Casa Leiria.

Stroud, C. B., Wilson, S., Durbin, E. C., \& Mendelsohn, K. A. (2011). Spillover to triadic and dyadic system in families with young children. Jornal of Family Psychology, 25(6), 919-930. doi:10.1037/a0025443

Teixeira, M. A. P., Oliveira, A. M., \& Wottrich, S.H. (2006). Escalas de práticas parentais (EPP): Avaliando dimensões de práticas parentais em relação a adolescentes. Psicologia: Reflexão e Crítica, 19(3), 433-441. doi:10.1590/S010279722006000300012
Wagner, A., \& Mosmann, C. (2009). A promoção da qualidade conjugal como estratégia de proteção dos filhos. In T. Féres-Carneiro (Eds.), Casal e Família: Permanências e rupturas (pp. 169189). São Paulo, SP: Casa do Psicólogo.

Zahn-Waxler, C., Shirtcliff, E. A., \& Marceau, K. (2008). Disorders of childhood and adolescence: Gender and psychopathology. Annual Review of Clinical Psychology, 4, 275-303. doi:10.1146/ annurev.clinpsy.3.022806.091358

Recebido: 20/05/2016

$1^{a}$ revisão: 02/03/2017

Aceite final: 10/03/2017

(C) $\mathrm{O}(\mathrm{s})$ autor(es), 2018. Acesso aberto. Este artigo está distribuído nos termos da Licença Internacional Creative Commons Atribuição 4.0 (http://creativecommons.org/licenses/by/4.0/), que permite o uso, distribuição e reprodução sem restrições em qualquer meio, desde que você dê crédito apropriado ao(s) autor(es) original(ais) e à fonte, fornecer um link para a licença Creative Commons e indicar se as alterações foram feitas. 